\title{
Existence of viscosity multi-valued solutions with asymptotic behavior for Hessian equations
}

\author{
Xianyu Meng and Yongqiang Fu*
}

\section{"Correspondence:}

fuyongqiang@hit.edu.cn Department of Mathematics, Harbin Institute of Technology, Harbin, 150001, P.R. China

\begin{abstract}
The Perron method is used to establish the existence of viscosity multi-valued solutions for a class of Hessian-type equations with prescribed behavior at infinity.
\end{abstract}

Keywords: Hessian equation; multi-valued solution; asymptotic behavior

\section{Introduction}

In [1, 2], the multi-valued solutions of the eikonal equation were studied. Later, in [3, 4] Jin et al. provided a level set method for the computation of multi-valued geometric solutions to general quasilinear partial differential equations and multi-valued physical observables to the semiclassical limit of the Schrödinger equations. In [5], Caffarelli and Li investigated the multi-valued solutions of the Monge-Ampère equation where they first introduced the geometric situation of the multi-valued solutions and obtained the existence, regularity and the asymptotic behavior at infinity of the multi-valued viscosity solutions. In [6] Ferrer et al. used complex variable methods to study the multi-valued solutions for the Dirichlet problems of Monge-Ampère equations on exterior planar domains. Recently, Bao and Dai discussed the multi-valued solutions of Hessian equations, see $[7,8]$. Motivated by the above works, in this paper we study the viscosity multi-valued solutions of the Hessian equation

$$
F\left(\lambda\left(D^{2} u\right)\right)=\sigma>0
$$

where $\sigma$ is a constant and $\lambda\left(D^{2} u\right)=\left(\lambda_{1}, \lambda_{2}, \ldots, \lambda_{n}\right)$ are eigenvalues of the Hessian matrix $D^{2} u$. $F$ is assumed to be defined in the symmetric open convex cone $\Gamma$, with vertex at the origin, containing

$$
\Gamma^{+}=\left\{\lambda \in \mathbf{R}^{n}: \text { each component of } \lambda, \lambda_{i}>0, i=1,2, \ldots, n\right\},
$$

satisfies the fundamental structure conditions

$$
F_{i}(\lambda)=\frac{\partial F}{\partial \lambda_{i}}>0 \quad \text { in } \Gamma, 1 \leq i \leq n,
$$


and $F$ is a continuous concave function. In addition, $F$ will be assumed to satisfy some more technical assumptions such as

$$
F>0 \quad \text { in } \Gamma, \quad F=0 \quad \text { on } \partial \Gamma \text {, }
$$

and for any $r \geq 1, R>0$,

$$
F\left(R\left(\frac{1}{r^{n-1}}, r, \ldots, r\right)\right) \geq F(R(1,1, \ldots, 1)) .
$$

For every $C>0$ and every compact set $K$ in $\Gamma$, there is $\Lambda=\Lambda(C, K)$ such that

$$
F(\Lambda \lambda) \geq C \quad \text { for all } \lambda \in K \text {. }
$$

There exists a number $\Lambda$ sufficiently large such that at every point $x \in \partial \Omega$, if $x_{1}, \ldots, x_{n-1}$ represent the principal curvatures of $\partial \Omega$, then

$$
\left(x_{1}, \ldots, x_{n-1}, \Lambda\right) \in \Gamma .
$$

Inequality (1.4) is satisfied by each $k$ th root of an elementary symmetric function $(1 \leq$ $k \leq n)$ and the $(k-l)$ th root of each quotient of the $k$ th elementary symmetric function and the $l$ th elementary symmetric function $(1 \leq l<k \leq n)$.

\section{Preliminaries}

The geometric situation of the multi-valued function is given in [5]. Let $n \geq 2, D \subset \mathbf{R}^{n}$ be a bounded domain with smooth boundary $\partial D$, and let $\Sigma \subset D$ be homeomorphic in $\mathbf{R}^{n}$ to an $n-1$ dimensional closed disc. $\partial \Sigma$ is homeomorphic to an $n-2$ dimensional sphere for $n \geq 3$.

Let $\mathbf{Z}$ be the set of integers and $M=(D \backslash \partial \Sigma) \times \mathbf{Z}$ denote a covering of $D \backslash \partial \Sigma$ with the following standard parametrization: fixing $x^{*} \in D \backslash \partial \Sigma$ and connecting $x^{*}$ by a smooth curve in $D \backslash \partial \Sigma$ to a point $x$ in $D \backslash \partial \Sigma$. If the curve goes through $\Sigma m \geq 0$ times in the positive direction (fixing such a direction), then we arrive at $(x, m)$ in $M$. If the curve goes through $\Sigma m \geq 0$ times in the negative direction, then we arrive at $(x,-m)$ in $M$.

For $k=2,3, \ldots$, we introduce an equivalence relation ' $\sim k$ ' on $M$ as follows: $(x, m)$ and $(y, j)$ in $M$ are ' $\sim k$ ' equivalent if $x=y$ and $m-j$ is an integer multiple of $k$. We let $M_{k}=$ $M / \sim k$ denote the $k$-sheet cover of $D \backslash \partial \Sigma$, and let $\partial^{\prime} M_{k}=\bigcup_{m=1}^{k}(\partial D \times\{m\})$.

We define a distance in $M_{k}$ as follows: for any $(x, m),(y, j) \in M_{k}$, let $l((x, m),(y, j))$ denote a smooth curve in $M_{k}$ which connects $(x, m)$ and $(y, j)$, and let $|l((x, m),(y, j))|$ denote its length. Define

$$
d((x, m),(y, j))=\inf _{l}|l((x, m),(y, j))|,
$$

where the infimum is taken over all smooth curves connecting $(x, m)$ and $(y, j)$. Then $d((x, m),(y, j))$ is a distance.

Definition 2.1 We say that a function $u$ is continuous at $(x, m)$ in $M_{k}$ if

$$
\lim _{d((x, m),(y, j)) \rightarrow 0} u(y, j)=u(x, m),
$$

and $u \in C^{0}\left(M_{k}\right)$ if for any $(x, m), u$ is continuous at $(x, m)$. 
Similarly, we can define $u \in C^{\alpha}\left(M_{k}\right), C^{0,1}\left(M_{k}\right)$ and $C^{2}\left(M_{k}\right)$.

Definition 2.2 A function $u \in C^{2}\left(M_{k}\right)$ is called admissible if $\lambda \in \bar{\Gamma}$, where $\lambda=\lambda\left(D^{2} u(x\right.$, $m))=\left(\lambda_{1}, \lambda_{2}, \ldots, \lambda_{n}\right)$ are the eigenvalues of the Hessian matrix $D^{2} u(x, m)$.

Definition 2.3 A function $u \in C^{0}\left(M_{k}\right)$ is called a viscosity subsolution (resp. supersolution) to (1.1) if for any $(y, m) \in M_{k}$ and $\xi \in C^{2}\left(M_{k}\right)$ satisfying

$$
u(x, m) \leq(\text { resp. } \geq) \xi(x, m), \quad(x, m) \in M_{k} \quad \text { and } \quad u(y, m)=\xi(y, m),
$$

we have

$$
F\left(\lambda\left(D^{2} \xi(y, m)\right)\right) \geq(\text { resp. } \leq) \sigma
$$

Definition 2.4 A function $u \in C^{0}\left(M_{k}\right)$ is called a viscosity solution to (1.1) if it is both a viscosity subsolution and a viscosity supersolution to (1.1).

Definition 2.5 A function $u \in C^{0}\left(M_{k}\right)$ is called admissible if for any $(y, m) \in M_{k}$ and any function $\xi \in C^{2}\left(M_{k}\right)$ satisfying $u(x, m) \leq(\geq) \xi(x, m), x \in M_{k}, u(y, m)=\xi(y, m)$, we have $\lambda\left(D^{2} \xi(y, m)\right) \in F$.

Remark It is obvious that if $u$ is a viscosity subsolution, then $u$ is admissible.

Lemma 2.1 Let $\Omega$ be a bounded strictly convex domain in $\mathbf{R}^{n}, \partial \Omega \in C^{2}, \varphi \in C^{2}(\bar{\Omega})$. Then there exists a constant $C$ only dependent on $n, \varphi$ and $\Omega$ such that for any $\xi \in \partial \Omega$, there exists $\bar{x}(\xi) \in \mathbf{R}^{n}$ such that

$$
|\bar{x}(\xi)| \leq C, \quad w_{\xi}(x)<\varphi(x) \quad \text { for } x \in \bar{\Omega} \backslash\{\xi\},
$$

where $w_{\xi}(x)=\varphi(\xi)+\frac{\bar{R}}{2}\left(|x-\bar{x}(\xi)|^{2}-|\xi-\bar{x}(\xi)|^{2}\right)$ for $x \in \mathbf{R}^{n}$ and $\bar{R}$ is a constant satisfying $F(\bar{R}, \bar{R}, \ldots, \bar{R})=\sigma$.

This is a modification of Lemma 5.1 in [5].

Lemma 2.2 Let $\Omega$ be a domain in $\mathbf{R}^{n}$ and $f \in C^{0}\left(\mathbf{R}^{n}\right)$ be nonnegative. Assume that the admissible functions $v \in C^{0}(\bar{\Omega}), u \in C^{0}\left(\mathbf{R}^{n}\right)$ satisfy, respectively,

$$
\begin{aligned}
& F\left(\lambda\left(D^{2} v\right)\right) \geq f(x), \quad x \in \Omega, \\
& F\left(\lambda\left(D^{2} u\right)\right) \geq f(x), \quad x \in \mathbf{R}^{n} .
\end{aligned}
$$

Moreover,

$$
\begin{aligned}
& u \leq v, \quad x \in \bar{\Omega}, \\
& u=v, \quad x \in \partial \Omega .
\end{aligned}
$$

Set

$$
w(x)= \begin{cases}v(x), & x \in \Omega, \\ u(x), & x \in \mathbf{R}^{n} \backslash \Omega .\end{cases}
$$


Then $w \in C^{0}\left(\mathbf{R}^{n}\right)$ is an admissible function and satisfies in the viscosity sense

$$
F\left(\lambda\left(D^{2} w(x)\right)\right) \geq f(x), \quad x \in \mathbf{R}^{n} .
$$

Lemma 2.3 Let $B$ be a ball in $\mathbf{R}^{n}$ and let $f \in C^{0, \alpha}(\bar{B})$ be positive. Suppose that $\underline{u} \in C^{0}(\bar{B})$ satisfies in the viscosity sense

$$
F\left(\lambda\left(D^{2} u\right)\right) \geq f(x), \quad x \in B
$$

Then the Dirichlet problem

$$
\begin{aligned}
& F\left(\lambda\left(D^{2} u\right)\right)=f(x), \quad x \in B, \\
& u=\underline{u}(x), \quad x \in \partial B
\end{aligned}
$$

admits a unique admissible viscosity solution $u \in C^{0}(\bar{B})$.

We refer to [9] for the proof of Lemmas 2.2 and 2.3.

\section{Existence of viscosity multi-valued solutions with asymptotic behavior}

In this section, we establish the existence of viscosity multi-valued solutions with prescribed asymptotic behavior at infinity of (1.1). Let $\Omega$ be a bounded strictly convex domain with smooth boundary $\partial \Omega$. Let $\Sigma$, diffeomorphic to an $(n-1)$-disc, be the intersection of $\Omega$ any hyperplane in $\mathbf{R}^{n}$. Let $M=\left(\mathbf{R}^{n} \backslash \partial \Sigma\right) \times \mathbf{Z}, M_{k}=M / \sim k$ be covering spaces of $\mathbf{R}^{n} \backslash \partial \Sigma$ as in Section 2. $\Sigma$ divides $\Omega$ into two open parts, denoted as $\Omega^{+}$and $\Omega^{-}$. Fixing $x^{*} \in \Omega^{-}$, we use the convention that going through $\Sigma$ from $\Omega^{-}$to $\Omega^{+}$denotes the positive direction through $\Sigma$. Our main result is the following theorem.

Theorem 3.1 Let $k \geq 3$. Then, for any $C_{m} \in \mathbf{R}$, there exists an admissible viscosity solution $u \in C^{0}\left(M_{k}\right)$ of

$$
F\left(\lambda\left(D^{2} u\right)\right)=\sigma, \quad(x, m) \in M_{k}
$$

satisfying

$$
\limsup _{|x| \rightarrow \infty}|x|^{n-2}\left|u(x, m)-\left(\frac{\bar{R}}{2}|x|^{2}+C_{m}\right)\right|<+\infty
$$

where $\bar{R}$ is a constant satisfying $F(\bar{R}, \bar{R}, \ldots, \bar{R})=\sigma$.

When

$$
F\left(\lambda\left(D^{2} u\right)\right)=\sigma_{k}\left(\lambda\left(D^{2} u\right)\right), \quad \Gamma=\Gamma_{k}=\left\{\lambda \in \mathbf{R}^{n}: \sigma_{j}>0, j=1,2, \ldots, k\right\},
$$

where the $k$ th elementary symmetric function

$$
\sigma_{k}(\lambda)=\sum_{i_{1}<\cdots<i_{k}} \lambda_{i_{1}} \cdots \lambda_{i_{k}}
$$

for $\lambda=\left(\lambda_{1}, \ldots, \lambda_{n}\right)$, in [8] Dai obtained the following result. 
Theorem 3.2 Let $k \geq 3$. Then, for any $C_{m} \in \mathbf{R}$, there exists a $k$-convex viscosity solution $u \in C^{0}\left(M_{k}\right)$ of

$$
\sigma_{k}\left(\lambda\left(D^{2} u\right)\right)=1, \quad(x, m) \in M_{k}
$$

satisfying

$$
\limsup _{|x| \rightarrow \infty}\left(|x|^{k-2}\left|u(x, m)-\left(\frac{C_{*}}{2}|x|^{2}+C_{m}\right)\right|\right)<\infty,
$$

where $C_{*}=\left(\frac{1}{C_{n}^{k}}\right)^{\frac{1}{k}}$.

Proof of Theorem 3.1 We divide the proof of Theorem 3.1 into two steps.

Step 1. By [10], there is an admissible solution $\Phi \in C^{\infty}(\bar{\Omega})$ of the Dirichlet problem:

$$
\begin{aligned}
& F\left(\lambda\left(D^{2} \Phi\right)\right)=C_{0}>\sigma, \quad x \in \Omega, \\
& \Phi=0, \quad x \in \partial \Omega .
\end{aligned}
$$

By the comparison principles in [11], $\Phi \leq 0$ in $\Omega$. Further, by Lemma 2.1, for each $\xi \in \partial \Omega$, there exists $\bar{x}(\xi) \in \mathbf{R}^{n}$ such that

$$
W_{\xi}(x)<\Phi(x), \quad x \in \bar{\Omega} \backslash\{\xi\},
$$

where

$$
W_{\xi}(x)=\frac{\bar{R}}{2}\left(|x-\bar{x}(\xi)|^{2}-|\xi-\bar{x}(\xi)|^{2}\right), \quad \xi \in \mathbf{R}^{n},
$$

and $\sup _{\xi \in \partial \Omega}|\bar{x}(\xi)|<\infty$. Therefore

$$
\begin{aligned}
& W_{\xi}(\xi)=0, \quad W_{\xi}(x) \leq \Phi(x) \leq 0, \quad x \in \bar{\Omega}, \\
& F\left(\lambda\left(D^{2} W_{\xi}(x)\right)\right)=F(\bar{R}, \bar{R}, \ldots, \bar{R})=\sigma, \quad \xi \in \mathbf{R}^{n} .
\end{aligned}
$$

Denote

$$
W(x)=\sup _{\xi \in \partial \Omega} W_{\xi}(x) .
$$

Then

$$
W(x) \leq \Phi(x), \quad x \in \Omega,
$$

and by $[12]$

$$
F\left(\lambda\left(D^{2} W\right)\right) \geq \sigma, \quad x \in \mathbf{R}^{n} .
$$

Define

$$
V(x)= \begin{cases}\Phi(x), & x \in \Omega, \\ W(x), & x \in \mathbf{R}^{n} \backslash \Omega .\end{cases}
$$


Then $V \in C^{0}\left(\mathbf{R}^{n}\right)$ is an admissible viscosity solution of

$$
F\left(\lambda\left(D^{2} V\right)\right) \geq \sigma, \quad x \in \mathbf{R}^{n} .
$$

Fix some $R_{1}>0$ such that $\bar{\Omega} \subset B_{R_{1}}(0)$, where $B_{R_{1}}(0)$ is the ball centered at the origin with radius $R_{1}$.

Let $R_{2}=2 R_{1} \bar{R}^{\frac{1}{2}}$. For $a>1$, defuse

$$
W_{a}(x)=\inf _{B_{R_{1}}} V+\int_{2 R_{2}}^{\left|\bar{R}^{\frac{1}{2}} x\right|}\left(s^{n}+a\right)^{\frac{1}{n}} d s, \quad x \in \mathbf{R}^{n} .
$$

Then

$$
D_{i j} W_{a}=\left(|y|^{n}+a\right)^{\frac{1}{n}-1}\left[\left(|y|^{n-1}+\frac{a}{|y|}\right) \bar{R} \delta_{i j}-\frac{a \bar{R}^{2} x_{i} x_{j}}{|y|^{3}}\right], \quad|x|>0,
$$

where $y=\bar{R}^{\frac{1}{2}} x$. By rotating the coordinates, we may set $x=(r, 0, \ldots, 0)$. Therefore

$$
D^{2} W_{a}=\left(R^{n}+a\right)^{\frac{1}{n}-1} \bar{R} \operatorname{diag}\left(R^{n-1}, R^{n-1}+\frac{a}{R}, \ldots, R^{n-1}+\frac{a}{R}\right),
$$

where $R=|y|$. Consequently, $\lambda\left(D^{2} W_{a}\right) \in \Gamma$ for $|x|>0$ and by (1.4)

$$
F\left(\lambda\left(D^{2} W_{a}\right)\right) \geq F(\bar{R}, \bar{R}, \ldots, \bar{R})=\sigma, \quad|x|>0 .
$$

Moreover,

$$
W_{a}(x) \leq V(x), \quad|x| \leq R_{1} .
$$

Fix some $R_{3}>3 R_{2}$ satisfying

$$
R_{3} \bar{R}^{\frac{1}{2}}>3 R_{2}
$$

We choose $a_{1}>1$ such that for $a \geq a_{1}$,

$$
W_{a}(x)>\inf _{B_{R_{1}}} V+\int_{2 R_{2}}^{3 R_{2}}\left(s^{n}+a\right)^{\frac{1}{n}} d s \geq V(x), \quad|x|=R_{3} .
$$

Then by (3.3) $R_{3} \geq R_{1}$. According to the definition of $W_{a}$,

$$
\begin{aligned}
W_{a}(x)= & \inf _{B_{R_{1}}} V+\int_{2 R_{2}}^{\left|\bar{R}^{\frac{1}{2}} x\right|} s\left(\left(1+\frac{a}{s^{n}}\right)^{\frac{1}{n}}-1\right) d s+\int_{2 R_{2}}^{\left|\bar{R}^{\frac{1}{2}} x\right|} s d s \\
= & \frac{\bar{R}}{2}|x|^{2}+C_{m}+\inf _{B_{R_{1}}} V+\int_{2 R_{2}}^{+\infty} s\left(\left(1+\frac{a}{s^{n}}\right)^{\frac{1}{n}}-1\right) d s-C_{m} \\
& -2 R_{2}^{2}-\int_{\left|\bar{R}^{2} x\right|}^{+\infty} s\left(\left(1+\frac{a}{s^{n}}\right)^{\frac{1}{n}}-1\right) d s, \quad x \in \mathbf{R}^{n} .
\end{aligned}
$$


Let

$$
\mu(m, a)=\inf _{B_{R_{1}}} V+\int_{2 R_{2}}^{+\infty} s\left(\left(1+\frac{a}{s^{n}}\right)^{\frac{1}{n}}-1\right) d s-C_{m}-2 R_{2}^{2}
$$

Then $\mu(m, a)$ is continuous and monotonic increasing for $a$ and when $a \rightarrow \infty, \mu(m, a) \rightarrow$ $\infty, 1 \leq m \leq k$. Moreover,

$$
W_{a}(x)=\frac{\bar{R}}{2}|x|^{2}+C_{m}+\mu(m, a)-O\left(|x|^{2-n}\right), \quad \text { when }|x| \rightarrow \infty .
$$

Define, for $a \geq a_{1}$ and $1 \leq m \leq k$,

$$
\underline{u}_{m, a}(x)= \begin{cases}\max \left\{V(x), W_{a}(x)\right\}-\mu(m, a), & |x| \leq R_{3}, \\ W_{a}-\mu(m, a), & |x| \geq R_{3} .\end{cases}
$$

Then by (3.4), for $1 \leq m \leq k$,

$$
\underline{u}_{m, a}(x)=\frac{\bar{R}}{2}|x|^{2}+C_{m}-O\left(|x|^{2-n}\right), \quad \text { when }|x| \rightarrow \infty,
$$

and by the definition of $V$,

$$
\underline{u}_{m, a}(x)=-\mu(m, a), \quad x \in \partial \Sigma .
$$

Choose $a_{2} \geq a_{1}$ large enough such that when $a \geq a_{2}$,

$$
\begin{aligned}
V(x)-\mu(m, a) & =V(x)-\inf _{B_{R_{1}}} V-\int_{2 R_{2}}^{+\infty} s\left(\left(1+\frac{a}{s^{n}}\right)^{\frac{1}{n}}-1\right) d s+C_{m}+2 R_{2}^{2} \\
& \leq C_{m} \\
& \leq \frac{\bar{R}}{2}|x|^{2}+C_{m}, \quad|x| \leq R_{3} .
\end{aligned}
$$

Therefore

$$
\underline{u}_{m, a}(x) \leq \frac{\bar{R}}{2}|x|^{2}+C_{m}, \quad a \geq a_{2}, x \in \mathbf{R}^{n} .
$$

By Lemma 2.2, $\underline{u}_{m, a} \in C^{0}\left(\mathbf{R}^{n}\right)$ is admissible and satisfies in the viscosity sense

$$
F\left(\lambda\left(D^{2} \underline{u}_{m, a}\right)\right) \geq \sigma, \quad x \in \mathbf{R}^{n} .
$$

It is easy to see that there exists a continuous function $a^{(m)}(a)$ such that $\lim _{a \rightarrow \infty} a^{(m)}(a)=$ $\infty$ and $\mu\left(m, a^{(m)}(a)\right)=\mu(1, a)$ for $2 \leq m \leq k$. So there exists $a_{3} \geq a_{2}$ such that $a^{(m)}(a)>a_{2}$ whenever $a \geq a_{3}$ and $2 \leq m \leq k$. Let $a^{(1)}(a)=a$ and define

$$
\underline{u}_{a}(x, m)=\underline{u}_{m, a^{(m)}(a)}(x), \quad(x, m) \in M_{k} .
$$


Then, by the definition of $\underline{u}_{m, a}$, when $a \geq a_{3}, \underline{u}_{a} \in C^{0}\left(M_{k}\right)$ is a locally admissible function satisfying

$$
\begin{aligned}
& \underline{u}_{a}(x, m)=\frac{\bar{R}}{2}|x|^{2}+C_{m}-O\left(|x|^{2-n}\right), \quad \text { when }|x| \rightarrow \infty, \\
& \underline{u}_{a}(x, m) \leq \frac{\bar{R}}{2}|x|^{2}+C_{m}, \quad x \in \mathbf{R}^{n}, 1 \leq m \leq k, \\
& \lim _{x \rightarrow \bar{x}} \underline{u}_{a}(x, m)=-\mu(1, a), \quad \bar{x} \in \partial \Sigma, 1 \leq m \leq k,
\end{aligned}
$$

and in the viscosity sense

$$
F\left(\lambda\left(D^{2} \underline{u}_{a}\right)\right) \geq \sigma, \quad(x, m) \in M_{k}
$$

Step 2. We define the solution of (3.1) by the Perron method.

For $a \geq a_{3}$, let $S_{a}$ denote the set of admissible functions $V \in C^{0}\left(M_{k}\right)$ which can be extended to $\partial \Sigma$ and satisfies

$$
\begin{aligned}
& F\left(\lambda\left(D^{2} V\right)\right) \geq \sigma, \quad(x, m) \in M_{k}, \\
& \lim _{x \rightarrow \bar{x}} V(x, m) \leq-\mu(1, a), \quad \bar{x} \in \Gamma, \\
& V(x, m) \leq \frac{\bar{R}}{2}|x|^{2}+C_{m}, \quad x \in \mathbf{R}^{n}, 1 \leq m \leq k .
\end{aligned}
$$

It is obvious that $\underline{u}_{a} \in S_{a}$. Hence $S_{a} \neq \emptyset$. Define

$$
u_{a}(x, m)=\sup \left\{V(x, m): V \in S_{a}\right\}, \quad(x, m) \in M_{k} .
$$

Next we prove that $u_{a}$ is a viscosity solution of (3.1). From the definition of $u_{a}$, it is a viscosity subsolution of (3.1) and satisfies

$$
u_{a}(x, m) \leq \frac{\bar{R}}{2}|x|^{2}+C_{m}, \quad x \in \mathbf{R}^{n} .
$$

So we need only to prove that $u_{a}$ is a viscosity supersolution of (3.1) satisfying (3.2).

For any $x_{0} \in \mathbf{R}^{n} \backslash \partial \Sigma$, fix $\varepsilon>0$ such that $\bar{B}=\overline{B_{\varepsilon}\left(x_{0}\right)} \subset \mathbf{R}^{n} \backslash \partial \Sigma$. Then the lifting of $B$ into $M_{k}$ is the $k$ disjoint balls denoted as $\left\{B^{(i)}\right\}_{i=1}^{k}$. For any $(x, m) \in B^{(i)}$, by Lemma 2.3 , there exists an admissible viscosity solution $\widetilde{u} \in C^{0}\left(\overline{B^{(i)}}\right)$ to the Dirichlet problem

$$
\begin{aligned}
& F\left(\lambda\left(D^{2} \widetilde{u}\right)\right)=\sigma, \quad(x, m) \in B^{(i)}, \\
& \tilde{u}=u_{a}, \quad(x, m) \in \partial B^{(i)} .
\end{aligned}
$$

By the comparison principle in [11],

$$
u_{a} \leq \widetilde{u}, \quad(x, m) \in B^{(i)} .
$$

Define

$$
\psi(x, m)= \begin{cases}\tilde{u}(x, m), & (x, m) \in B^{(i)}, \\ u_{a}(x, m), & (x, m) \in M_{k} \backslash\left\{B^{(i)}\right\}_{i=1}^{k} .\end{cases}
$$


By Lemma 2.2,

$$
F\left(\lambda\left(D^{2} \psi(x, m)\right)\right) \geq \sigma, \quad x \in \mathbf{R}^{n} .
$$

As

$$
\begin{aligned}
& F\left(\lambda\left(D^{2} \widetilde{u}\right)\right)=\sigma=F\left(\lambda\left(D^{2} g\right)\right), \quad(x, m) \in B^{(i)}, \\
& \tilde{u}=u_{a} \leq g, \quad(x, m) \in \partial B^{(i)},
\end{aligned}
$$

where $g(x, m)=\frac{\bar{R}}{2}|x|^{2}+C_{m}$, we have

$$
\widetilde{u} \leq g, \quad(x, m) \in \overline{B^{(i)}}
$$

by the comparison principle in [11]. Therefore $\psi \in S_{a}$.

By the definition of $u_{a}, u_{a} \geq \psi$ in $M_{k}$. Consequently, $\widetilde{u} \leq u_{a}$ in $B^{(i)}$ and further $\widetilde{u}=u_{a}$, $(x, m) \in B^{(i)}$ in view of (3.5). Since $x_{0}$ is arbitrary, we conclude that $u_{a}$ is an admissible viscosity solution of (3.1).

By the definition of $u_{a}$,

$$
\underline{u}_{a} \leq u_{a} \leq g, \quad(x, m) \in M_{k}
$$

so $u_{a}$ satisfies (3.2) and we complete the proof of Theorem 3.1.

\section{Competing interests}

The authors declare that they have no competing interests.

\section{Authors' contributions}

All authors contributed to each part of this work equally and read and approved the final version of the manuscript.

\section{Acknowledgements}

This work was supported by the National Natural Science Foundation of China (Grant No. 11371110).

Received: 5 March 2014 Accepted: 25 June 2014 Published online: 24 September 2014

\section{References}

1. Gosse, L, Jin, S, Li, X: Two moment systems for computing multiphase semiclassical limits of the Schrödinger equation. Math. Models Methods Appl. Sci. 13, 1689-1723 (2003)

2. Izumiya, S, Kossioris, GT, Makrakis, GN: Multivalued solutions to the eikonal equation in stratified media. Q. Appl. Math. 59, 365-390 (2001)

3. Jin, S, Osher, S: A level set method for the computation of multivalued solutions to quasi-linear hyperbolic PDEs and Hamilton-Jacobi equations. Commun. Math. Sci. 1, 575-591 (2003)

4. Jin, S, Liu, H, Osher, S, Tsai, Y: Computing multivalued physical observables for the semiclassical limit of the Schrödinger equation. J. Comput. Phys. 205, 222-241 (2005)

5. Caffarelli, L, Li, YY: Some multi-valued solutions to Monge-Ampère equations. Commun. Anal. Geom. 14, 411-441 (2006)

6. Ferrer, L, Martínez, A, Milán, F: An extension of a theorem by K. Jörgens and a maximum principle at infinity for parabolic affine spheres. Math. Z. 230, 471-486 (1999)

7. Dai, LM, Bao, JG: Multi-valued solutions to Hessian equations. Nonlinear Differ. Equ. Appl. 18, 447-457 (2011)

8. Dai, LM: Existence of multi-valued solutions with asymptotic behavior of Hessian equations. Nonlinear Anal. 74, 3261-3268 (2011)

9. Tian, BP, Fu, YQ: Existence of viscosity solutions for Hessian equations in exterior domains. Front. Math. China 9, 201-211 (2014)

10. Caffarelli, L, Nirenberg, L, Spruck, J: The Dirichlet problem for nonlinear second-order elliptic equations III: functions of eigenvalues of the Hessians. Acta Math. 15, 261-301 (1985)

11. Trudinger, NS: The Dirichlet problem for the prescribed curvature equations. Arch. Ration. Mech. Anal. 111, 153-179 (1990)

12. Ishii, H, Lions, PL: Viscosity solutions of fully nonlinear second-order elliptic partial differential equations. J. Differ. Equ. 83, 26-78 (1990) 
doi:10.1186/s13661-014-0165-8

Cite this article as: Meng and Fu: Existence of viscosity multi-valued solutions with asymptotic behavior for Hessian equations. Boundary Value Problems 2014 2014:165.

Submit your manuscript to a SpringerOpen ${ }^{\circ}$ journal and benefit from:

- Convenient online submission

- Rigorous peer review

- Immediate publication on acceptance

Open access: articles freely available online

- High visibility within the field

- Retaining the copyright to your article

Submit your next manuscript at $\boldsymbol{s p r i n g e r o p e n . c o m ~}$ 